\title{
Chemopreventative Potential of Plant-Based Extracts Coffee (Coffea) and Mauby (Colubrina Arborescens) using the Global Antioxidant Response (GAR) Method via in vitro Simulated Digestion
}

\author{
Hannah Gross ${ }^{1}$, Cheryl Rock ${ }^{1}$, Yada Treesukosol ${ }^{2} \&$ Virginia Gray $^{1}$ \\ ${ }^{1}$ Department of Family and Consumer Sciences, California State University, Long Beach, 90840, USA \\ ${ }^{2}$ Department of Psychology, California State University Long Beach, Long Beach, CA 90840, USA \\ Correspondence: Dr. Cheryl Rock, 1250 Bellflower Boulevard, Long Beach, CA 90840, USA. Tel: \\ 562-985-4497. E-mail: cheryl.rock@csulb.edu
}

Received: February 18, 2021

Accepted: May 21, $2021 \quad$ Online Published: May 25, 2021

doi:10.5539/jfr.v10n3p43

URL: https://doi.org/10.5539/jfr.v10n3p43

\begin{abstract}
Plant-based extracts such as coffee (coffea) and Mauby (Colubrina Arborescens) were tested for chemopreventative potential by measuring their antioxidant activity (i.e., reducing power and free radical scavenging capacity) conventionally using chemical assays 1, 1-Diphenyl-2-picryl-hydrazyl (DPPH) and Ferric Reducing Antioxidant Power (FRAP). Alternately, extracts were also analyzed for their chemopreventative potential via a novel method (Global Antioxidant Response [GAR]), where they were subjected to simulated digestion before their antioxidant activity was determined via conventional methods previously mentioned. Next, the antioxidant capacities of the extracts, conventional versus the novel (GAR) method were compared. Overall, the analysis indicated that the reducing power (FRAP) and free radical scavenging capacity (DPPH) of coffee and Mauby were reduced $(\sim 16 \%)$ after undergoing simulated digestion. It was also observed that while the antioxidants in Mauby scavenged radicals at a significantly higher capacity than those in coffee $(95.7 \% \pm 0.67$ and $90 \% \pm 2.1$ before digestion, and $77.7 \% \pm 2.2$ and $74.6 \% \pm 2.3$ after digestion, respectively), antioxidants in coffee exhibited higher reducing power compared to those in Mauby. Specifically, after undergoing simulated digestion, $1.12 \mathrm{mM} \mathrm{FeSO} 4 / \mathrm{mL} \pm 0.05$ to $0.68 \mathrm{mM} \mathrm{FeSO} / \mathrm{mL} \pm 0.07$ ions were reduced before digestion, and $0.73 \mathrm{mM} \mathrm{FeSO} / \mathrm{mL} \pm 0.09$ to $0.48 \mathrm{mM} \mathrm{FeSO} 4 / \mathrm{mL} \pm 0.04$ ions were reduced after digestion for coffee and Mauby, respectively. These findings suggest that while the antioxidants in coffee may have been more powerful in their ability to reduce ions, the antioxidants in Mauby may have been more effective in scavenging and neutralizing radicals.
\end{abstract}

Keywords: chemoprevention, antioxidants, phytonutrients, coffee, mauby

\section{Introduction}

\subsection{Problem of Study and Importance}

Briefly, chemoprevention refers to the use of medicine, vitamins, or other dietary agents (usually plant-based) to reduce cancer risk or slow its development (National Institutes of Health [NIH], 2019). Specifically, the use of agents (i.e., bioactive compounds) as a means of chemoprevention may serve as a valuable method in preventing the growth and development of cancer. In particular, the objectives of chemoprevention can be classified as three different levels (i.e., primary, secondary, or tertiary), depending on the stage of intervention. According to the literature (Veronesi \& Bonnani, 2006), the goal of primary chemoprevention is to prevent the onset of a disease, whereas as secondary chemoprevention is designed to treat a population with a premalignant condition, and last, tertiary chemoprevention intends to protect individuals who were previously treated for cancer and may still be at risk. In brief, chemoprevention may serve as a valuable intervention strategy against the burden of cancer as it aims to prevent, reduce, and slow the risk of cancer development. Specifically, this research focuses on the chemopreventative potential of two plant extracts, coffee and Mauby. While coffee is widely consumed in the US, the use of barks, such as Mauby, has demonstrated a recent increase in commercial interest and may serve as a potential untapped source of beneficial antioxidants when consumed. Therefore, this study aims to also explore the chemoprotective properties of a lesser known agent and possible opportunity for market expansion. 


\subsection{Relationship of our Study to Previous Work in the Area}

While there is ample research investigating associations of plant extracts and reduction of disease risk, there are conflicting conclusions regarding their overall chemopreventative potential using conventional methods (i.e., soley chemical assays). To be specific, much of the literature regarding antioxidant compounds in plant-based extracts uses conventional chemical assays as a means of measuring antioxidant compounds. Therefore, using novel methods such as the Global Antioxidant Response (GAR) method would more accurately represent the behaviour regarding antioxidant capacity in a biological environment. Specifically, the GAR method simulates gastric digestion, which may provide more insight into what changes in the bioactivity of antioxidants are likely to occur in the human gut under physiological conditions. As highlighted by Perez-Burillo et al. (2018), most methods of measuring antioxidant capacities rely on the use of organic solvents which differ greatly from those found within the human body; therefore, a more physiologic approach would be to use an in vitro process which more accurately represents the digestion process taking place within the gut. With over 606,000 estimated cancer-related deaths in 2019 (American Cancer Society [ACS], 2019), chemoprevention research is especially important because it may provide more insight into new information regarding the mechanisms of plant-based extracts and their chemopreventative potential under simulated biological conditions. Additionally, the use and consumption of plant extracts from novel sources such as Mauby Bark in comparison to those commonly consumed (e.g., coffee) have not been extensively studied. To summarize, studies comparing the antioxidant activity of plant-based extracts in a simulated digestion environment is very limited, justifying the need for more research in this area.

\subsection{Hypotheses}

In this study, it was hypothesized that there would be no significant difference $(\mathrm{p}<0.05)$ in antioxidant activity between the coffee and Mauby extract when using conventional antioxidant assays DPPH and FRAP. Additionally, it was hypothesized that there would be no significant difference $(p<0.05)$ in measured antioxidant capacity of the plant extracts after undergoing the GAR method when compared to the measured antioxidant capacities when using the conventional assays alone. In short, the overall objective of this study was to evaluate the chemopreventative potential of plant-based extracts, namely coffee and Mauby, before and after in vitro simulated digestion by comparing their antioxidant activity. Antioxidant levels were measured using the conventional chemical assays DPPH and FRAP, and the GAR method was used in order to simulate the digestion process. According to literature, the GAR method serves as an optimization to the limitations of the conventional chemical assays and other methods of measuring antioxidant capacities. For example, a study by Pastoriza et al. (2011) found the GAR method to more effectively measure total antioxidant capacity when compared to two other methods, the chemical extraction and Quencher methods. Therefore, by comparing the measured capacities of the extracts conventionally versus the novel GAR method, it provides a better understanding of the chemopreventative potential of these plant extracts under simulated digestive conditions.

\subsection{Relationship of Hypotheses and Research Design}

The hypotheses of our study relate to the comparative research design in that they seek to compare antioxidant capacities between the sample beverage extracts- both before and after undergoing simulated digestion- using the two chemical assays DPPH and FRAP for comparison. The plant sample extracts mentioned in the hypotheses contain phytochemicals which may serve as blocking agents of carcinogenesis in that they prevent DNA mutations in the body (Landis-Piwowar \& Lyer, 2014). To elaborate, the antioxidants in the phytochemicals can potentially prevent cell damage caused by free radicals and oxidized ions within the body, which in this experiment are represented by DPPH radicals and ferric ions. Furthermore, the GAR method represents the phases of human digestion in which these phytochemicals would be subjected to when consumed. In brief, the use of the DPPH and FRAP assays allows for comparison of antioxidant activity before and after undergoing simulated digestion, providing some understanding of how these antioxidants serve as blocking agents in the development of cancer.

\subsection{Theoretical and Practical Implications of the Study}

Experimentally, plant-based materials when consumed in the diet are known to contain beneficial compounds such as phytochemicals that have been implicated in some studies regarding chemoprevention. For example, a study by Ciaramelli et al. (2019) collected coffee samples from 10 different countries to be compared for antioxidant activity and analyzed for implications on human health. Specifically, they found that the Arabica coffee species contained the highest amounts of beneficial phytochemicals trigonelline, choline, and chlorogenic acids. These chlorogenic acids include radical scavenging properties (Niseteo et al., 2012) as well as anti-cancer, immunoprotective, cardiovascular protective, anti-hypertensive, anti-inflammatory, anti-viral, and anti-fungal 
properties (Palmioli et al., 2017). Additionally, a meta-analysis by Gan et al. (2017) found that individuals consuming four or more cups of coffee per day had a 7\% decrease in risk of developing colon cancer, although these associations were not observed with rectal cancer. Overall, higher intakes of coffee were inversely associated with risk of colorectal cancer, suggesting potential associations between colorectal cancer risk and coffee consumption. A separate study by Embola et al. (2019) assessed antioxidant activity of Mauby and found the bark to contain polyphenols which exhibited free radical scavenging activity, suggesting their potential to protect against oxidative stress within the body. As can be seen, these results are suggestive of the multitude of diseases in which plant extracts may offer protection from.

In regards to practical implications, an increase in the use of barks in the United States may offer expansions in product development and functional food applications. As suggested in the literature, widely consumed coffee contains antioxidant compounds, as does Mauby, a lesser-known and potentially untapped source of these beneficial compounds. While coffee is largely consumed in the United States, the consumption and use of barks is far less widespread but could be a resourceful option as the entire Colubrina Arborescens plant can be utilized to serve a variety of purposes. Furthermore, there has been a recent emergence of products and elixirs becoming commercially available which contain Mauby and other barks, and the use of these barks may serve a variety of health benefits as they contain beneficial phytonutrients similar to those of coffee. This increase in consumer interest for bark products warrants expanded research on the health implications of consuming these products. In summary, this research aims to compare the chemopreventative potential of commonly consumed coffee to lesser consumed Mauby beverage, which may offer similar health benefits as well as the potential for market expansion.

\section{Method}

\subsection{Selection of the Sample}

Quality samples of Mauby bark and coffee were obtained from reputable third party vendors.

\subsection{The GAR Method}

As previously mentioned, the GAR method serves as a simulated digestion process, which includes three total phases in order to represent the main phases of human digestion: (1) oral, (2) gastric, and (3) intestinal. After both the coffee and Mauby samples were subjected to all 3 phases, respectively, the antioxidant activities of the digested extracts were evaluated. The GAR technique was carried out in accordance with the procedure described by Perez-Burillo et al. (2018), with some modifications. Specifically, the following method is an extension of a previous GAR technique as described by Minekus et al. (2014). First, extracts were prepared and brewed in accordance with standard brewing techniques for each of these extracts in order to be representative of how the typical consumer would prepare to ingest these beverages. Specifically, the coffee was brewed using a standard drip coffee maker, using $28 \mathrm{~g}$ of coffee grinds and $355 \mathrm{~mL}$ of deionized distilled water $\left(\mathrm{DDH}_{2} \mathrm{O}\right)$. Conversely, Mauby was brewed in accordance with the Embola study. Specifically, $20 \mathrm{~g}$ of Mauby was brewed in $250 \mathrm{~mL}$ boiling water at $100^{\circ} \mathrm{C}$ for 30 minutes. Next, the extracts were each separately combined with simulated salivary fluid (SSF), simulated gastric fluid (SGF), and simulated intestinal fluid (SIF) to undergo simulated digestion using the following procedures:

(1) Oral Phase: for this phase, $5 \mathrm{~mL}$ of extract were combined with $5 \mathrm{~mL}$ of SSF with alpha-amylase, and $25 \mu 1$ of $\mathrm{CaCl}_{2}$. The mixture was then incubated at $37^{\circ} \mathrm{C}$ for 2 minutes to allow for simulated salivary digestion.

(2) Gastric Phase: for this phase, the mixture from the oral phase was then combined with $10 \mathrm{~mL}$ of SGF with pepsin and $5 \mu \mathrm{l}$ of $\mathrm{CaCl}_{2}$, as well as $1 \mathrm{~N} \mathrm{HCl}$ in order to lower the $\mathrm{pH}$ to 3.0. To allow for simulated gastric digestion, the mixture was then incubated again at $37^{\circ} \mathrm{C}$ for the duration of 2 hours.

(3) Intestinal Phase: for this phase, the same sample was then combined with $20 \mathrm{~mL}$ of SIF with pancreatin and bile salts, and $40 \mathrm{~mL}$ of $\mathrm{CaCl}_{2}$. The $\mathrm{pH}$ was then adjusted to 7.0 by adding $1 \mathrm{~N} \mathrm{NaOH}$. The mixture was incubated at $37^{\circ} \mathrm{C}$ for 2 hours.

After incubation, the test tube containing the digested extracts was placed into an ice bath in order to stop any further enzymatic activity. Moreover, the extracts were centrifuged for 10 minutes at $6000 \mathrm{rpm}$ at $4^{\circ} \mathrm{C}$ using a Thermo Scientific Sorvall ST 8 R centrifuge. Last, the antioxidant capacity of each of the digested extracts were assessed using the assays DPPH and FRAP.

\subsection{Determination of the Ferric Reducing Antioxidant Power (FRAP)}

The antioxidant power of the extracts was measured using the FRAP assay in the procedure as described by Abbasian et al. (2013). First, ferrous sulfate $\left(\mathrm{FeSO}_{4}\right)$ standards $(0,3,6,9$, and $12 \mathrm{mM})$ were prepared to generate 
a standard curve (Figure 1) which was used to determine the antioxidant power of the plant extracts. Next, the FRAP reagent was prepared using 3 reagents combined in the following 10:1:1 ratio: $300 \mathrm{mM} / \mathrm{L}$ of acetate buffer (pH 3.6), $10 \mathrm{mM} / \mathrm{L}$ of 2,4,6- tripryridyl-s-triazine (TPTZ) dissolved in $40 \mathrm{mM} / \mathrm{L}$ hydrochloric acid (HCL), and $20 \mathrm{mM}$ of ferric chloride $\left(\mathrm{FeCl}_{3}\right)$ dissolved in $\mathrm{DDH}_{2} \mathrm{O}$. To follow, $3.8 \mathrm{~mL}$ of the FRAP reagent was added to 200 $\mu \mathrm{L}$ of the plant extract in dark test tubes which were then incubated at $37^{\circ} \mathrm{C}$ for 5 minutes. Last, the absorbance of the sample was used to determine the total antioxidant power of the reagents using a UV spectrometer (Thermo Scientific AquaMate $8000 \mathrm{UV}$-Vis Spectrophotometer) at a wavelength of $593 \mathrm{~nm}$. The results were expressed as $\mathrm{mM} \mathrm{FeSO}_{4} / \mathrm{mL}$. The extracts were analyzed in triplicate and averaged for analysis.

FRAP Standard Curve Data

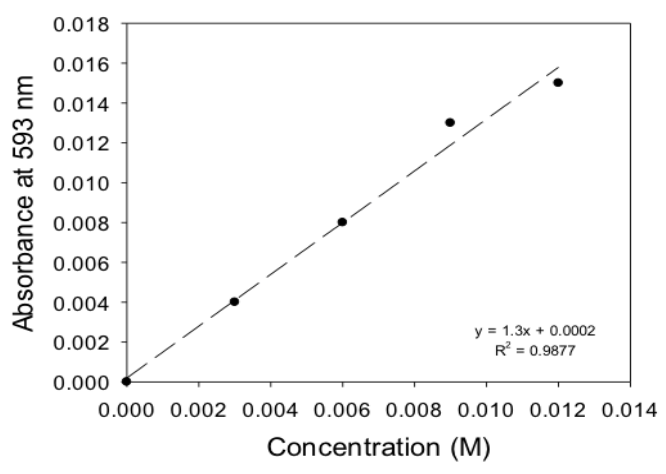

Figure 1. Standard Curve for FRAP Reagent

\subsection{Determination of the Free Radical Scavenging Activity (DPPH)}

The free radical scavenging activity of the plant extracts was measured with the radical DPPH (1, 1-Diphenyl-2-picryl-hydrazyl), using the following method as described by Priyanka et al. (2013). First, $0.1 \mathrm{mM}$ $\mathrm{DPPH} \bullet$ was dissolved with $90 \%$ methanol in a beaker protected from light. Next, $3 \mathrm{~mL}$ of the DPPH• solution was combined with $1 \mathrm{~mL}$ of the plant sample solution. Next, a control was also prepared using $1 \mathrm{~mL}$ of $90 \%$ methanol and $3 \mathrm{~mL}$ of $\mathrm{DPPH} \bullet$ solution. Then, the solution was mixed and incubated in a dark cabinet for 30 minutes. Lastly, the solution was analyzed using a UV spectrometer (Thermo Scientific Aquamate 8000 UV-Vis Spectrophotometer) at a wavelength of $517 \mathrm{~nm}$. Free radical scavenging ability was calculated as the percentage of free radicals scavenged by the plant extract, using Equation 1. Each of the samples were analyzed in triplicate and results were averaged for analysis. Before analyzing data, both the DPPH and FRAP chemical assays were carried out on all samples for coffee and Mauby, including samples which were subjected to simulated digestion as well as samples which were not subjected to simulated digestion.

$$
\% \text { Inhibition of DPPH } \bullet \text { Activity }=\left(\mathrm{A}_{\text {control }}-\mathrm{A}_{\text {sample }} / \mathrm{A}_{\text {control }}\right) \times 100
$$

\subsection{Data Analysis}

Data were analyzed using the Statistical Package for Social Sciences (SPSS) version 26. For the first hypothesis, an independent samples t-test was performed separately for both of the chemical assays DPPH and FRAP, in order to compare the antioxidant capacity of coffee to Mauby extract when using the conventional chemical assays. For the second hypothesis, an independent samples t-test was performed in order to compare the measured antioxidant capacity for both the coffee and Mauby extracts after undergoing simulated digestion via the GAR method. Additionally, a two-way ANOVA comparing DPPH values for coffee and Mauby when using the conventional method versus using the GAR method was performed. The two-way ANOVA was also performed to compare FRAP values for coffee and Mauby when using the conventional and the GAR method. Furthermore, post-hoc two-sample t-tests were used. A value of $p<0.05$ was considered statistically significant.

\section{Results and Discussion}

\subsection{Radical-Scavenging Ability of Coffee and Mauby}

Antioxidant activity of coffee and Mauby was measured using both the DPPH and FRAP chemical assays. It was hypothesized that there would be no significant difference $(\mathrm{p}<0.05)$ in antioxidant activity between the coffee extract and Mauby extract when using conventional antioxidant assays DPPH and FRAP. It was also hypothesized that there would be no significant difference $(\mathrm{p}<0.05)$ in measured antioxidant capacity of the plant extracts after undergoing the GAR method when compared to the measured antioxidant capacities when 
using the conventional assays alone.

The DPPH assay was used to determine the free radical-scavenging ability of the plant samples, as measured by the percentage of DPPH radicals scavenged by the samples within a 30-minute period. When using the DPPH assay, results showed Mauby to be more effective than coffee in scavenging free radicals. Specifically, the ability of coffee to scavenge the DPPH radicals was found to be an average of $90 \%( \pm 2.1)$, while the ability of the Mauby to scavenge DPPH radicals was an average of $95.7 \%( \pm 0.67)$. In other words, Mauby scavenged significantly more free radicals than coffee.

The radical-scavenging ability of the plant samples was measured once again after undergoing a simulated digestion process using the GAR method. Analysis indicated that Mauby scavenged significantly more DPPH radicals compared to coffee. Specifically, Mauby scavenged $77.7 \%( \pm 2.2)$, while coffee scavenged $74.6 \%( \pm 2.3)$. Results revealed that less radical-scavenging ability was measured for both the coffee and Mauby extracts after undergoing the GAR method. A two-way ANOVA (method x stimulus) was used to compare the method of the conventional DPPH assay alone versus the DPPH assay in combination with the novel GAR method with coffee and mauby. A two-way ANOVA revealed a main effect of method $(F(1,28)=1421.373, p<0.001)$, main effect of stimulus $(\mathrm{F}(1,28)=65.224, \mathrm{p}<0.001)$ and interaction effect $(\mathrm{F}(1,28)=9.102, \mathrm{p}=0.005)$. These comparisons also survived Bonferroni corrections, both when using the chemical assay DPPH alone, and when using DPPH with GAR, $(\mathrm{t}(28)=-10.140$, corrected $\mathrm{p}<0.001)$, and $(\mathrm{t}(28)=-3.764$, corrected $\mathrm{p}=0.003)$, respectively. Coffee and Mauby's effectiveness in scavenging the DPPH radicals decreased by $15.4 \%$ and $18 \%$, respectively. This observed difference in radical-scavenging ability suggests that the antioxidants in both plant samples were less effective after being exposed to the environment of the mouth, stomach, and intestine (Figure 2).

The previously mentioned Embola study (2019) also investigated the antioxidant potential of Mauby bark using the DPPH assay. Analysis indicated that after being brewed for 30 minutes, the Mauby bark was able to scavenge $75 \%$ of the DPPH radicals. After 45 minutes, $81 \%$ of radicals were scavenged, and after 60 minutes, $83 \%$ of DPPH radicals were scavenged. These findings suggest that the effectiveness of the antioxidants in Mauby increased as brewing time was increased, potentially due to the extract being more concentrated. These results are consistent with the present findings in that Mauby was able to scavenge around $78 \%$ of DPPH radicals even after undergoing simulated digestion. It is interesting, however, that in the present study, Mauby was able to scavenge close to $96 \%$ of the radicals after 30 minutes, whereas in the Embola study, $75 \%$ of the radicals were scavenged after the same amount of time. These differences could potentially be attributed to the use of the more novel GAR method in addition to the DPPH assay in the current study. A separate study by Wolska et al. (2017) compared the radical scavenging activity of several varieties of brewed coffee using the DPPH assay and found DPPH inhibition to be $71.97-83.21 \%$ for all varieties. These findings are similar to that of the present study in which brewed coffee was able to scavenge $74.6 \%$ of radicals even after undergoing simulated digestion.

It should also be noted that in the present study, in accordance with standard preparation times for these beverages, coffee was brewed for 5 minutes while Mauby was brewed for 30 minutes. The purpose of the difference in preparation times was in order to most accurately reflect what consumers would be drinking when ingesting these beverages. Although the coffee and Mauby samples have different brewing times, this study aimed to realistically capture the antioxidant capacity of these plant sample extracts in the state in which they are meant to be consumed.

\section{DPPH Mean Antioxidant Values}

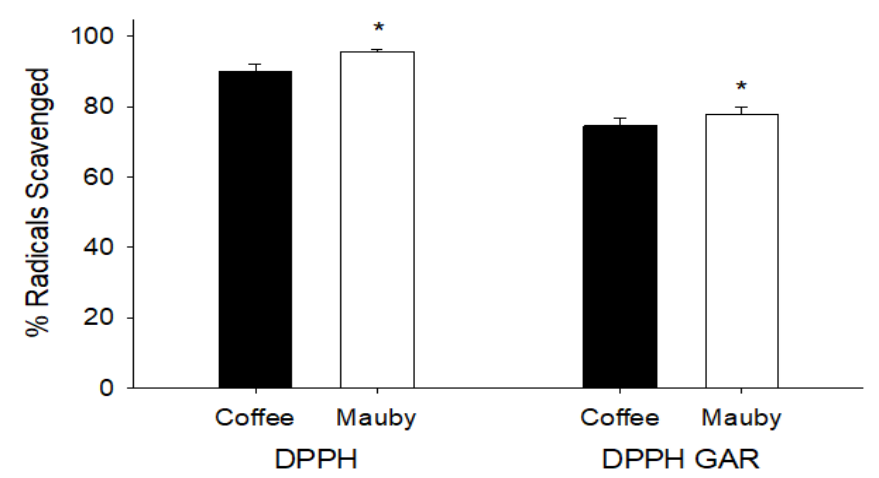

Figure 2. Average DPPH values for coffee versus Mauby using the conventional versus GAR method

\footnotetext{
* indicates a significant difference between coffee and Mauby
} 


\subsection{Ferric Reducing Antioxidant Power of Coffee and Mauby}

The FRAP assay was used to determine the reducing ability or power of the plant samples by measuring the amount of ions reduced from the ferric $\left(\mathrm{Fe}^{3+}\right)$ to the ferrous $\left(\mathrm{Fe}^{2+}\right)$ form, as expressed in $\mathrm{mM} \mathrm{FeSO} / \mathrm{mL}$. The analysis found that the antioxidants in coffee were able to reduce a higher amount of ferric ions $(1.12 \mathrm{mM}$ $\left.\mathrm{FeSO}_{4} / \mathrm{mL}[ \pm 0.04]\right)$ in comparison to those in Mauby $\left(0.68 \mathrm{mM} \mathrm{FeSO}_{4} / \mathrm{mL}[ \pm 0.07]\right)$, suggesting that coffee was 1.6 times more powerful in ion reduction. The hypothesis stating that there would be no significant difference between the coffee extract and Mauby extract when using conventional antioxidant assays DPPH and FRAP, was therefore rejected.

The ability of the plant samples to reduce ferric $\left(\mathrm{Fe}^{3+}\right)$ ions to their ferrous $\left(\mathrm{Fe}^{2+}\right)$ form was then measured again using the FRAP chemical assay after the samples had undergone simulated digestion using the GAR method. Analysis indicated that after simulated digestion, the reducing power of coffee was 1.5 times more powerful than that of Mauby $\left(0.73 \mathrm{mM} \mathrm{FeSO} / 4 \mathrm{~mL}[ \pm 0.09]\right.$ and $0.48 \mathrm{mM} \mathrm{FeSO}_{4} / \mathrm{mL}[ \pm 0.04]$, respectively). There was less ion-reducing ability measured for both the coffee and the Mauby extracts after undergoing the GAR method. A two-way ANOVA (method $x$ stimulus) was used to compare the method of the conventional FRAP assay alone versus the FRAP assay in combination with the novel GAR method with coffee and Mauby. The analysis revealed a main effect of method $(F(1,28)=377.999, p<0.001)$, main effect of stimulus $(F(1,28)=419.840$, $\mathrm{p}<0.001)$ and interaction $\mathrm{F}(1,28)=40.525, \mathrm{p}<0.001$. These comparisons also survived Bonferroni corrections, both when using the FRAP assay alone, and when using FRAP with GAR, $(\mathrm{t}(28)=22.811, \mathrm{p}<0.001$, corrected $\mathrm{p}<0.001)$, and $(\mathrm{t}(28)=9.533, \mathrm{p}<0.001$, corrected $\mathrm{p}<0.001)$, respectively. To specify, after undergoing simulated digestion, the amount of ions reduced by the antioxidants in coffee and Mauby were decreased by $0.39 \mathrm{mM}$ $\mathrm{FeSO}_{4} / \mathrm{mL}$ and $0.2 \mathrm{mM} \mathrm{FeSO}_{4} / \mathrm{mL}$, respectively. Similar to the DPPH assay, the findings could be related to the antioxidants' ability to withstand the environment of the simulated digestive system. Therefore, the hypothesis stating that there would be no significant difference in measured antioxidant capacity of the plant extracts after undergoing the GAR method when compared to the measured antioxidant capacities when using the conventional assays alone was also rejected (Figure 3).

The previously mentioned Embola study also measured the reducing power of Mauby bark extract using the FRAP chemical assay. Analysis indicated that after 30 and 45 minutes of brew time, the FRAP values for Mauby ranged from 6.29 to $6.90 \mathrm{mM} \mathrm{FeSO}_{4} / \mathrm{mL}$. These findings from the Embola study are substantially higher than the results from the current study. As mentioned previously, the incorporation of the more novel GAR method could possibly account for these differences. While there are many studies quantifying the reducing power of coffee beverages and coffee grounds, few studies express these results in $\mathrm{mM} \mathrm{FeSO} / \mathrm{mL}$, therefore making them difficult to compare to the present study. One related study, however, assessed the antioxidant capacity of phenolic compounds from spent coffee grounds using the FRAP assay, and found them to range from 0.040 to $0.102 \mathrm{mM} \mathrm{Fe}$ (II)/g of sample (Mussatto et al., 2011). Although the study investigates spent coffee grounds rather than brewed coffee, it still provides some insight into the antioxidant potential of the specific phenolic compounds found within coffee, and these results are similar to those reported in the present study. Additionally, a study by Martinez-Ruiz et al. (2018), evaluated the FRAP activity of brewed coffee after several time periods, and found it to have FRAP values of $1.29 \mathrm{mg} / \mathrm{mL}$ after less than 30 minutes of brew time. These findings are very similar to that of the present study, which found coffee to have FRAP activity of $1.12 \mathrm{mM} \mathrm{FeSO}_{4} / \mathrm{mL}$ after 5 minutes of brew time. After undergoing simulated digestion, however, the coffee samples in the present study were found to have a decreased FRAP value, most likely due to undergoing the process of simulated digestion.

FRAP Mean Antioxidant Values

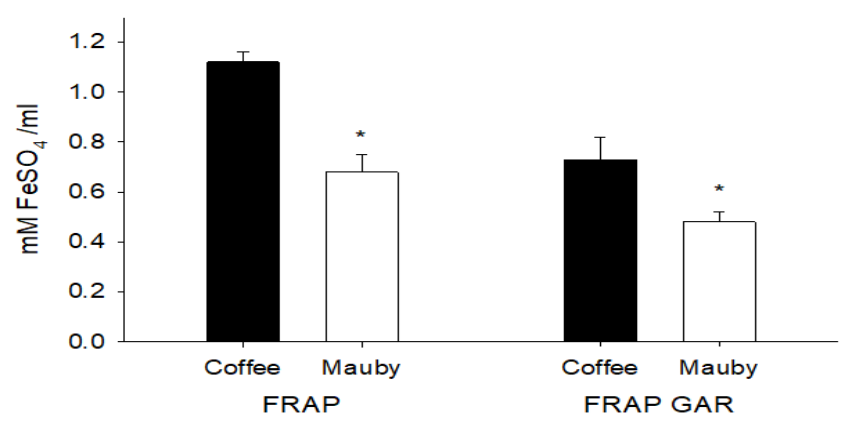

Figure 3. Average FRAP values for coffee versus Mauby using the conventional versus GAR method

* indicates a significant difference between coffee and Mauby 


\section{Conclusion}

To summarize, the antioxidant activity of plant extracts coffee and Mauby were evaluated using conventional chemical assays and compared to a novel GAR method in order to measure their antioxidant capacity as an indicator of chemopreventative potential under simulated biological conditions. Overall, the findings suggest that the antioxidants in coffee were more powerful in their ability to reduce ions compared to those in Mauby, while antioxidants in Mauby were found to be more effective in scavenging free radicals. Regardless, coffee may offer chemoprotective properties due to its potential power in reducing ions- potentially damaging ones- within the body when consumed. In regards to Mauby, its antioxidants were found to be less powerful (as demonstrated by FRAP values), however more effective (as demonstrated by DPPH values), in their ability to actually scavenge and neutralize radicals, also suggesting some chemopreventative properties. Thus, an increase in the use of barks could offer potential benefits to consumers. An especially interesting concept derived from these results is that while coffee was found to be more powerful (as demonstrated by its FRAP values), it was not found to be more effective (based off its DPPH values), suggesting that the power of an antioxidant does not determine whether or not that antioxidant will actually be effective in scavenging radicals. In other words, it may be beneficial to consume a wide variety of antioxidant-containing plant foods in order to increase the possibility of consuming antioxidants which are both powerful and effective. Furthermore, future research regarding ways in which to maximize both effectiveness and power of antioxidants would be useful. Limitations of this study are that this research was conducted in vitro; therefore observations made may not be representative of what actually occurs during human digestion of these products. Future research involving in vivo methods may provide more insight into these findings. In conclusion, the consumption of coffee and Mauby, along with a variety of other plant foods in the diet, may offer chemoprotective properties due to their antioxidants' potential in scavenging and neutralizing damaging free radicals within the body.

\section{Acknowledgements}

Dr. Cheryl Rock and Hannah Gross adapted and conducted the experiment and collected data. Hannah Gross, Dr. Cheryl Rock, and Dr. Yada Treesukosol analyzed and interpreted data. Hannah Gross wrote the manuscript, while Dr. Cheryl Rock, Dr. Yada Treesukosol, and Dr. Virginia Gray participated in drafting and editing the manuscript.

\section{References}

Abbasian, S., Karimi, F., Moghaddam, G., Soroush, A., Moloudian, H., \& Ahosseini, M. S. (2013). Antioxidant properties of different black tea samples and some Iranian native plants. Pharmacie Globale, 4(2), 1-5.

American Cancer Society. (2019). Cancer facts and figures. Retrieved from https://www.cancer.org/content/dam/cancer-org/research/cancer-facts-and-statistics/annual-cancer-facts-and -figures/2019/cancer-facts-and-figures-2019.pdf

Ciaramelli, C., Palmioli, A., \& Airoldi, C. (2019). Coffee variety, origin and extraction procedure: implications for coffee beneficial effects on human health. Food Chemistry, 278, 47-55. https://doi.org/10.1016/j.foodchem.2018.11.063

Embola, J., Rock, C., Wang, L., Reiboldt, W., Ahmed, S., \& Aliabadi, S. (2019). An assessment of total polyphenolic content and antioxidant potential of mauby bark extracts (colubrina arborescens) brewed for different lengths of time. Journal of Food Research, 8(3). https://doi.org/10.5539/jfr.v8n3p26

Gan, Y., Wu, J., Zhang, S., Li, L., Cao, S., Mkandawire, N., ... Lu, Z. (2017). Association of coffee consumption with risk of colorectal cancer: a meta-analysis of prospective cohort studies. Oncotarget, 8(12), 18699-18711. https://doi.org/10.18632/oncotarget

Landis-Piwowar, K. R., \& Lyer, N. R. (2014). Cancer chemoprevention: current state of the art. Cancer Growth and Metastasis, 7, 19-25. https://doi.org/10.4137/CGM.S11288

Martinez-Ruiz, C., Lozano, G., Roldan-Cruz, C., Meraz, M., \& Rodriguez-Huezo, M. E. (2018). Evolution of antioxidant activity in heated coffee brew. Revista Mexicana de Ingeniería Química, 17(2), 613-619. https://doi.org/10.24275/uam/izt/dcbi/revmexingquim/2018v17n2/MartinezC

Minekus, M., Alminger, M., Alvito, P., Balance, S., Bohn, T., Bourlieu, C., ... Brodkorb, A. (2014). A standardised static in vitro digestion method suitable for food- an international consensus. Food \& Function, 5, 1113-1124. https://doi.org/10.1039/C3FO60702J

Mussatto, S., Ballesteros, L., Martins, S., \& Teixeira, J. (2011). Extraction of antioxidant phenolic compounds from spent coffee grounds. Separation and Purification Technology, 83(15), 173-179. 
https://doi.org/10.1016/j.seppur.2011.09.036

National Institutes of Health. (2019). NCI dictionary of cancer terms. Retrieved from https://www.cancer.gov/publications/dictionaries/cancer-terms

Niseteo, T., Komes, D., Belscak-Cvitanovic, A., Horzic, D., \& Budec, M. (2012). Bioactive composition and antioxidant potential of different commonly consumed coffee brews affected by their preparation technique and milk addition. Food Chemistry, 134(4), 1870-1877. https://doi.org/10.1016/j.foodchem.2012.03.095

Palmioli, A., Ciaramelli, C., Tisi, R., Spinelli, M., De Sanctis, G., Sacco, E., \& Airoldi, C. (2017). Natural compounds in cancer prevention: effects of coffee extracts and their main polyphenolic component 5-CQA on oncogenic ras proteins. Chemistry-An Asian Journal, 12(18), 2457-2466. https://doi.org/10.1002/asia.201700844

Pastoriza, S., Degaldo-Andrade, C., Haro, A., \& Rufian-Henares, J. A. (2011). A physiologic approach to test the global antioxidant response of foods: the GAR method. Food Chemistry, 129(4), 1926-1932. https://doi.org/10.1016/j.foodchem.2011.06.009

Perez-Burillo, S., Rufian-Henares, J. A., \& Pastoriza, S. (2018). Towards an improved global antioxidant response method (GAR+): physiological-resembling in vitro digestion-fermentation method. Food Chemistry, 239, 1253-1262. https://doi.org/10.1016/j.foodchem.2017.07.024

Priyanka, C., Kadam, D. A., Kadam, A. S., Ghule, Y. A., \& Aparadh, V. T. (2013). Free radical scavenging $(\mathrm{DPPH})$ and ferric reducing ability (FRAP) of some gymnosperm species. International Journal of Research in Botany, 3(2), 34-36. https://www.researchgate.net/publication/239949381

Veronesi, U., \& Bonnani, B. (2006). Chemoprevention: from research to clinical oncology. European Journal of Cancer, 41(13), 1833-1841. https://doi.org/10.1016/j.ejca.2005.06.007

Wolska, J., Janda, K., Jakubeyzk, K., Symkowiak, M., Chlubek, D., \& Gutowska, I. (2017). Levels of antioxidant activity and fluoride content in coffee infusion of arabica, robusta, and green coffee beans in according to their brewing methods. Biological Trace Element Research, 179, 327-333.

https://doi.org/10.1007/s12011-017-0963-9

\section{Copyrights}

Copyright for this article is retained by the author(s), with first publication rights granted to the journal.

This is an open-access article distributed under the terms and conditions of the Creative Commons Attribution license (http://creativecommons.org/licenses/by/4.0/). 